\title{
La percepción de sentido de la vida en el ciclo vital: una perspectiva temporal
}

\author{
The Perception of meaning of Life in Uital Cycle: 9 Time Perspective \\ f percepção de sentido de vida no ciclo vital: uma perspectiva temporal
}

\author{
Thiago Antonio Avellar De Aquino*, Valdiney Veloso Gouveia*, \\ Eliseudo Salvino Gomes**, Lorena Bandeira Melo de Sá*** \\ *Universidad Federal da Paraíba, **Universidad Católica de Brasília, \\ ***Universidad Estadual da Paraiba
}

Doi: http://dx.doi.org/10.12804/revistas.urosario.edu.co/apl/a.3728

\section{Resumen}

Este estudio ha tenido como objetivo conocer en qué medida la percepción ontológica del tiempo y la sensación de presencia y búsqueda de sentido cambian según el ciclo de la vida. Participaron 766 voluntarios, la mayoría mujeres (59\%), agrupados en los siguientes rangos: juventud ( $N=350 ; 18$ a 24 años), joven adulto ( $N=195 ; 25$ a 34 años), adulto $(N=110 ; 35$ a 59 años) y tercera edad ( $N=111 ; 60$ a 87 años). Los participantes contestaron al cuestionario sentido de la vida (CSV), la escala de percepción ontológica del tiempo (ЕРОT) y preguntas demográficas. Los resultados sugirieron que el sentido aumenta según las fases de la vida, en cuanto que la búsqueda de sentido disminuye. Se constató también que los adultos y las personas de tercera edad perciben mayor presencia de sentido en el presente cuando son comparados con los jóvenes; además, los promedios en la dimensión de futuro fueron menores en las fases más maduras. Se concluyó que los hallazgos fueron en la dirección de la literatura, sugiriendo implicaciones clínicas de este estudio.

Palabras clave: logoterapia, sentido de la vida, temporalidad, ciclo de vida.

\section{fbstract}

This study aimed to know to which extent the ontological perception of time, the sense of presence and the search for meaning change according to the life cycle. Participants were 766 volunteers, mostly women (59\%), grouped into the following stratifications: youth ( $N=350 ; 18$ to 24 years), young adult $(N=195 ; 25$ to 34 years), adult ( $N=110 ; 35$ to 59 years), and older

\footnotetext{
* Universidad Federal da Paraíba, João Pessoa, Brasil. Centro de Educación, Universidade Federal da Paraíba. Cidade Universitária, s/n - Castelo Branco. 58051-900 João Pessoa Brasil. Correos electrónicos: logosvitae@hotmail.com, Thiago.aquino@ pesquisador.cnpq.br

** Universidad Católica de Brasília, Brasília, Brasil.

*** Universidad Estadual da Paraíba, Campina Grande, Brasil.
}

Cómo citar este artículo: Aquino, T. A. A., Gouveia, V. V., Gomes, E. S., \& Melo de Sá, L. B. (2017). La percepción de sentido de la vida en el ciclo vital: una perspectiva temporal. Avances en Psicología Latinoamericana, 35(2), 375-386. doi: http:// dx.doi.org/10.12804/revistas.urosario.edu.co/apl/a.3728 
adult ( $N=111 ; 60$ to 87 years). They answered the Meaning of Life Questionnaire, the Ontological Perception of Time Scale and demographic questions. Results indicated that the presence for meaning increased according to the cycle life, meanwhile the search for meaning decreased. It has also observed that adults and older adults perceived greater presence of meaning in the present when compared to the young people; moreover, the mean scores on the future dimension were lowest among more maturity people. In conclusion, these findings were in line with the literature, suggesting clinical implications of this study.

Keywords: logotherapy, meaning of life, temporality, life cycle.

\section{Resumo}

Este estudo tem tido como objetivo conhecer em que medida a percepção ontológica do tempo e a sensação de presença e busca de sentido mudam segundo o ciclo de vida. Participaram 766 voluntários, a maioria mulheres (59\%), agrupados nos seguintes rangos: Juventude ( $N$ $=350 ; 18$ a 24 anos $)$, Jovem Adulto $(N=195 ; 25$ a 34 anos), Adulto ( $N=110 ; 35$ a 59 anos) e Terceira Idade $(N=111 ; 60$ a 87 anos). Os participantes contestaram ao Questionário Sentido de Vida (CSV), a Escala de Percepção Ontológica do Tempo (ЕРOT) e perguntas demográficas. Os resultados sugeriram que o sentido aumenta segundo as fases da vida, em quanto que a busca de sentido diminui. Constatou-se também que os adultos e as pessoas de terceira idade percebem maior presença de sentido no presente quando são comparados com os jovens; além disso, as médias na dimensão de futuro foram menores nas fases mais maduras. Concluiu-se que os resultados foram na direção da literatura, sugerindo implicações clínicas deste estudo.

Palavras-chave: logoterapia, sentido de vida, temporalidade, ciclo de vida.

\section{Introducción}

El presente estudio busca evidencias empíricas para el desarrollo de la percepción de sentido de la vida en el ciclo vital de la existencia humana. Frankl (1992), fundador de la teoría centrada en el sentido, no presupuso estadios de desarrollo de sentido en la vida, aunque reconozca que en todas las fases de la existencia, del joven adolescente a la persona de tercera edad, hay una preocupación del hombre en relación con el significado de su vida. Sin embargo, aunque en su obra no haya cualquier diferenciación entre el sentido de vida de adolescentes y adultos, por ejemplo, quizá este sentido se manifieste de forma distinta, careciendo de comprobación empírica. Precisamente, este aspecto motivó el presente estudio.

Teniendo en cuenta el análisis existencial de Viktor Frankl, se formularon dos preguntas orientadoras de esta investigación: ¿Cuál es el grado de presencia y búsqueda de sentido en las fases del desarrollo humano? Y ¿cómo las personas perciben ontológicamente la temporalidad (presente, pasado y futuro) en las fases de la vida? Procurando fundamentar estas preguntas, se revisan los conceptos básicos de la logoterapia, la teoría ontológica del tiempo y las fases de desarrollo humano.

\section{Logoterapia y análisis existencial}

La logoterapia puede ser comprendida como un sistema de psicoterapia que se fundamenta en una filosofía de la existencia. Sus fundamentos pueden ser sistematizados en tres instancias: la libertad de voluntad, la voluntad de sentido y el sentido de la vida.

La libertad de la voluntad es un axioma que predice que las personas actúan a partir de sus posibilidades y se posicionan ante las situaciones inmutables. De hecho, el hombre no es libre de condiciones biológicas, psicológicas y sociológicas, aunque es libre para posicionarse o actuar sobre las condiciones de la existencia, superándolas o resignándose a los condicionamientos, eligiendo dejarse determinar o actuar según un sentido o 
conjunto de valores (Frankl, 2000). Sin embargo, el hombre no es un ser que reacciona impulsado solamente por sus instintos y estímulos, sino que actúa en un mundo - es "un ser-en-el-mundo", en el decir de Heidegger, (1989)—, y este mundo es formado por otros seres y sentidos a los que él procura autotrascender (Frankl, 2003a). De hecho, Frankl (1990) entiende que el verdadero ser humano es el que siempre decide lo que es. Por lo tanto, todo hombre ha de buscar su misión, pues todo tiene un sentido y siempre se puede cambiar con libertad.

La voluntad de sentido es la motivación primaria del hombre. Las personas desean realizar sentidos en la vida y buscan descubrir valores dignos de vivir y de morir. Según Frankl (1992), la pregunta sobre el sentido de la vida emerge en la adolescencia con la maduración psicofísica de la persona. El hombre es atraído hacia los valores, no deseando solamente vivir; por ser un ente finito, emerge en él la voluntad de encontrar un sentido en el mundo. Frankl (2003b) describe que, en la actualidad, el hombre sufre de un vacío interior y un sentimiento de falta de sentido. No obstante, la frustración existencial no es una patología, salvo cuando está acompañada por rasgos neuróticos en la dimensión psicofísica.

Frankl $(1988,2003 a)$ describe que el vacío existencial es ocasionado por la pérdida del instinto y de la tradición, lo que ocasiona que el hombre actual no sepa lo que debe hacer. Los síntomas sociales del vacío existencial se manifiestan por medio de la drogadicción, agresión y depresión/suicidio. Como las manifestaciones de esa tríada se encuentran en todas las sociedades, Frankl supone una neurosis de masa, concluyendo que "vivimos en una época en la que predomina la sensación de una falta general de sentido" (Frankl, 2003b, p. 19). De este modo, lo que le falta a los hombres es justamente comprometerse con algo, su entrega a una misión existencial por la cual pueda decidirse libremente.

En la formulación de la logoterapia, Frankl (2003b) hace una diferenciación entre sentido y valores: el sentido es algo único en las situaciones en que el ser humano está inmerso, mientras que los valores son categorías universales de sentido, trascendiendo a las situaciones específicas. Por ese motivo, la vida permanece con sentido a pesar del desaparecimiento de las tradiciones. Según ese abordaje, hay tres vías valorativas para la realización de sentido: valores vivenciales (vivencias de personas u obras artísticas y naturaleza), valores creativos (laborar y crear algo para el mundo) y valores actitudinales (tomar posturas en situaciones inmutables y de sufrimiento).

El sentido de la vida es el presupuesto filosófico de la logoterapia. Indica que en la vida hay un sentido incondicional que necesita ser descubierto por el ser humano. Este sentido cambia de un momento al otro y de una persona a otra, no dependiendo de condiciones internas o externas. El sentido está en el mundo, en consecuencia, el hombre necesita trascender a sí mismo para algo (una causa) o alguien (un ser amado). Así, la logoterapia concibe el sentido de tres formas: sentido de la vida, sentido en la vida y sentido en el mundo. Hay también una relación temporal de esos conceptos, pues el sentido de la vida solo puede ser percibido en el final de la existencia del hombre, mientras el sentido en la vida puede ser descubierto en todos los momentos de la vida del ser; por fin, el sentido en el mundo (el cosmos) comprende una inquietud humana con respecto al sufrimiento humano, que justifica el propio sentido de la vida. De este modo, el sufrimiento sin sentido resulta en desespero o ausencia de sentido. En términos generales, Frankl (1992) se contrapone a las teorías escépticas, que resultan siempre en una cosmovisión nihilista, y salvaguarda la concepción de que el hombre es un ser en relación con los valores existenciales.

En resumen, el hombre busca realizar sentido en su vida por medio también de valores existenciales, reconociendo que es un ser finito y un ser para la muerte (Heidegger, 1989). Esa búsqueda no se restringe a los aspectos positivos ni a los trá- 
gicos (por ejemplo, dolor, sufrimiento y muerte), circunscritos en una condición temporal y espacial. Así resta, pues, considerar la teoría ontológica del tiempo, que se presenta a continuación.

\section{Teoría ontológica del tiempo}

La concepción del tiempo ha sido uno de los temas centrales desde la Antigüedad, considerando su medición, pasaje y fluidez. Frankl (1988) presenta una teoría ontológica del tiempo que considera la transitoriedad de la existencia, partiendo de dos aspectos: una crítica de las concepciones platónicas y del "trágico heroísmo" (existencialismo). El primer aspecto asevera que el futuro no existe, así como el pasado; de este modo, el tiempo es una apariencia, lo que sugiere que la división de la temporalidad en pasado, presente y futuro constituye una ilusión de la consciencia. El segundo aspecto indica que la persona "viene de la nada y vuelve a la nada", y, a pesar de eso, debe dar heroicamente sentido a la existencia.

El platonismo niega la existencia temporal y hace creer que todas las cosas ya están preestablecidas, puesto que el tiempo no es fluido, y ello resulta en un quietismo (Frankl, 1988). Platón en realidad considera el tiempo de origen cosmológico, y en este sentido Heidegger (1989) procura establecer la distinción entre el 'ser' y el 'no ser'. El mundo del 'ser' es fundamental y no está sujeto a mutaciones; el mundo es, según su pensamiento, eternamente el mismo. Este mundo, sin embargo, es el mundo de las ideas, aprehensible tan solo por la inteligencia y puede ser comprendido a partir de la razón. A su vez, Frankl (1989) discute sobre el existencialismo, su necesidad de considerar el aquí y ahora y sugiere un "pesimismo del presente". En contraposición a la perspectiva existencialista, este autor defiende que "el futuro ciertamente no es nada; pero el pasado es la pura verdad" (Frankl, 1988, p. 51).

Según se planteó previamente, la posición de Frankl parte de la transitoriedad de las situaciones, puesto que todo es fugaz. De este modo, concluye que el tiempo fluye del futuro para el pasado, de la nada (posibilidades) para el ser pasado (realidades) (Frankl, 1988; Lukas, 1992). Por lo tanto, el futuro es constituido por las posibilidades del ser, y así es denominado "de la nada". El pasado, por su vez, es el "ser", es decir, el pasado es formado por todos los valores realizados, no pudiendo ser eliminado del mundo. Por fin, el presente es definido como "un espacio limítrofe entre la nada y la existencia" (Frankl, 1988, p. 56), correspondiendo al espacio que hace del hombre un ser responsable por todo lo que hizo en el pasado.

Las conclusiones de la concepción de Frank1 se pueden resumir como siguen: (I) en la muerte el hombre no tiene la dimensión futura; (II) tiene solamente su ser pasado; (III) por consiguiente, el hombre es su propia historia (Frankl, 1988). En una perspectiva del desarrollo humano, la juventud tiene una amplitud del pasado muy pequeña, en comparación con su futuro (posibilidades abiertas). Por otro lado, en la tercera edad el hombre tiene un área de su "ser pasado" muy grande (por ejemplo, trabajos logrados, amor vivenciado y sufrimiento enfrentado con coraje), pero las posibilidades futuras son restrictas (Lukas, 1992).

Considerando lo comentado, la logoterapia asume una postura intermediaria entre el quietismo y el existencialismo, concibiendo el "activismo del futuro" y un "optimismo del pasado", puesto que el futuro será pasado y el pasado es la dimensión perene de la existencia humana. En términos generales, el hombre es un ser inacabado, que solo completa su esencia en el momento de su muerte, de modo que "el muerto es su propio pasado" (Frankl, 1988, p. 57). En consonancia con esta perspectiva, entiende este autor que el futuro es fluctuante, o sea, "es disponible para nosotros, podemos disponer de él, podemos cambiarlo y respectivamente cambiar nosotros mismos. Pero el pasado está fijo" (Frankl, 1988, p. 52). El tiempo, de este modo, no es un fenómeno constante, pero 
se vivencia de diversas maneras durante el desarrollo humano.

\section{Etapas del desarrollo humano}

Existen diversas definiciones de las etapas del desarrollo humano, siendo un ejemplo clásico la tipología presentada por Erikson (1998), teniendo en cuenta el abordaje psicoanalítico. Según Erikson (1987):

[...] todo ser vivo tiene un plano básico de desarrollo, y es a partir de este plano que se agregan las partes, teniendo cada una de ellas su propio tiempo de ascensión, maduración y ejercicio, hasta que todas hayan surgido para formar un todo en funcionamiento (p. 92).

Este principio, denominado de epigenético, se aplica a las personas en sus procesos biológicos (soma), psíquicos (organización de las experiencias) y sociales (ethos). A su vez, Levinson (1986) propone periodos de desarrollo universales, sugiriendo la siguiente secuencia: preadultez (desde el nacimiento a los 22 años), adultez temprana (de 17 a los 45 años), adultez media (de 40 a los 64 años), adultez tardía (de 60 a los 85 años) y adultez realmente tardía (de 80 años en adelante).

De hecho, es posible considerar diferentes clasificaciones de grupos etarios. En el presente estudio, considerando la concepción de Barrientos-Parra (2004), que indica la necesidad de respetar el contexto cultural y que no se contemplen unas categorías inmutables, una vez que los cambios en los rangos de edades reflejan procesos, se ha tenido en cuenta la siguiente clasificación: jóvenes (18 a los 24 años), adultos jóvenes (25 a los 34 años), adultos maduros (35 a los 59 años) y personas de la tercera edad (60 o más años). Los grupos de jóvenes y adultos jóvenes fueron elegidos teniendo en cuenta que comprenden fases importantes de la vida, configurando, respectivamente, la formación superior e inserción en el mercado de tra- bajo (Soares, Carvalho \& Kipnis, 2003). Según la persona esté en la segunda mitad de los 30 años, es común que procure afirmarse en la sociedad adulta, logrando la madurez a partir de la separación de su familia de origen, sea por matrimonio o con el fin de vivir solo (Agudo, 2008). Después de los 40 años de edad, la persona encuentra estabilidad en la vida, hasta los 60 años, cuando la disminución de su participación en el mercado de trabajo es evidente, sobre todo por empezar su proceso de jubilación, caracterizando una fase nueva en su vida (Raffa \& Bortolossi, 2007; World Health Organization, 1986).

Teniendo en cuenta esta clasificación de las fases de desarrollo, principalmente al considerar la relación del hombre y su inserción (y retirada) en el mercado de trabajo, admitiendo una percepción dinámica del sentido de la vida, que puede cambiar según el ciclo de vida, se ha pensado llevar a cabo el presente estudio. Específicamente, su objetivo ha sido conocer en qué medida la percepción ontológica del tiempo y la presencia y búsqueda de sentido cambian durante la vida.

\section{Método}

\section{Participantes}

Se ha considerado una muestra no-probabilística compuesta por 766 participantes brasileños que residían en la ciudad de João Pessoa (PB), incluyendo estudiantes universitarios y miembros de la población general. Estos fueron en su mayoría mujeres (59\%), distribuidos del siguiente modo con respecto al grupo etario: jóvenes $(N=350$; amplitud de 18 a 24 años, $M=20,8, D T=1,84)$, jóvenes adultos $(N=195$; amplitud de 25 a 34 años, $M=28,3, D T=2,66)$, adultos $(N=110$; amplitud de 35 a 59 años, $M=45,8, D T=7.16)$ y tercera edad $(N=111$; amplitud de 60 a 87 años, $M=65,6, D T=4,81)$.

\section{Instrumentos}


Además de preguntas demográficas (sexo y edad), los participantes han sido solicitados a contestar a los siguientes instrumentos:

Cuestionario sentido de la vida. Este instrumento fue adaptado al contexto brasileño, comprobando evidencias de su validez factorial y fiabilidad. De manera específica, Aquino et al. (2015) observaron una estructura con dos factores aceptables de esta medida $(G F I=0,94, A G F I=0,90, C F I=0,95$ y $R M S E A=0,086$ ); sus alfas de Cronbach fueron 0,89 (búsqueda de sentido) y 0,85 (presencia de sentido). Resultados similares han sido descritos con anterioridad por Steger, Frazier, Oishi y Kaler (2006), quienes también observaron dos factores: presencia de sentido y búsqueda de sentido. Este cuestionario está formado por diez ítems (por ejemplo, Comprendo el significado de mi vida; Estoy siempre buscando algo que haga que mi vida sea significativa), que son contestados en escala de 7 puntos, variando de 1 (totalmente falso) a 7 (totalmente verdadero).

Escala de percepción ontología del tiempo (ЕРOT). Teniendo por base la concepción de temporalidad de Frankl (1988, 1989) y Lukas (1990a), Aquino (2009) desarrolló esta medida. Su propósito ha sido evaluar la percepción del ser con respecto al pasado (al mirar el pasado: me siento realizado(a) por lo que ya he logrado en la vida), presente (al mirar el presente: veo siempre una razón para estar en el mundo) y futuro (al mirar el futuro: veo muchas posibilidades de elección), reuniendo tres ítems en cada dimensión temporal, además de un ítem que ofrece una evaluación global de la vida (índice de congruencia del self: admito que hay una gran distancia entre quien "soy" y quien "podría ser"). Los ítems son dispuestos en una escala de respuesta de 5 puntos, con los extremos 1 (totalmente en desacuerdo) y 5 (totalmente de acuerdo). Realizando un análisis factorial confirmatorio, este autor verificó índices de ajuste adecuados para la estructura trifactorial: $\chi^{2} / \mathrm{gl}=1,89$, RMSEA $=0,054, G F I=0,97, A G F I=$ 0,94 y $C F I=0,98$.

\section{Procedimiento}

La recolección de los datos se realizó en tres contextos: (i) en una universidad pública, (ii) en un grupo de convivencia para ancianos y (iii) en la población general. Tanto en aplicaciones individuales como colectivas, los instrumentos fueron respondidos de manera individual, siendo ofrecidas las mismas instrucciones a los encuestados, a saber: el carácter voluntario y confidencial de todas las informaciones, demandando inicialmente contar con la autorización de las personas que decidieron contestar y las entidades que los representaban. Además, fueron considerados todos los aspectos éticos de investigaciones con seres humanos, según determina el Consejo Nacional de Salud de Brasil (Resolución 466/12).

\section{Análisis de datos}

Para efectos de los análisis estadísticos, se elaboró una base de datos única en el paquete estadístico PASW (versión 18). Se han llevado a cabo cálculos de estadísticos descriptivos (promedio, desviación típica), correlación de Pearson y análisis de varianza (Anova), en este caso considerando el test post hoc de Bonferroni.

\section{Resultados}

Fueron efectuados análisis de varianzas (ANO$V A$ ), objetivando averiguar si hay diferencias en los puntajes promedios de los respondientes en las variables del estudio según los grupos de edad (juventud, joven adulto, adulto y tercera edad). Se han observado diferencias entre los promedios de los dos factores de sentido de la vida: presencia $(F=17,22, p<0,001)$ y búsqueda de sentido $(F=13,88, p<0,001)$. En la figura 1 se presenta la gráfica correspondiente. 


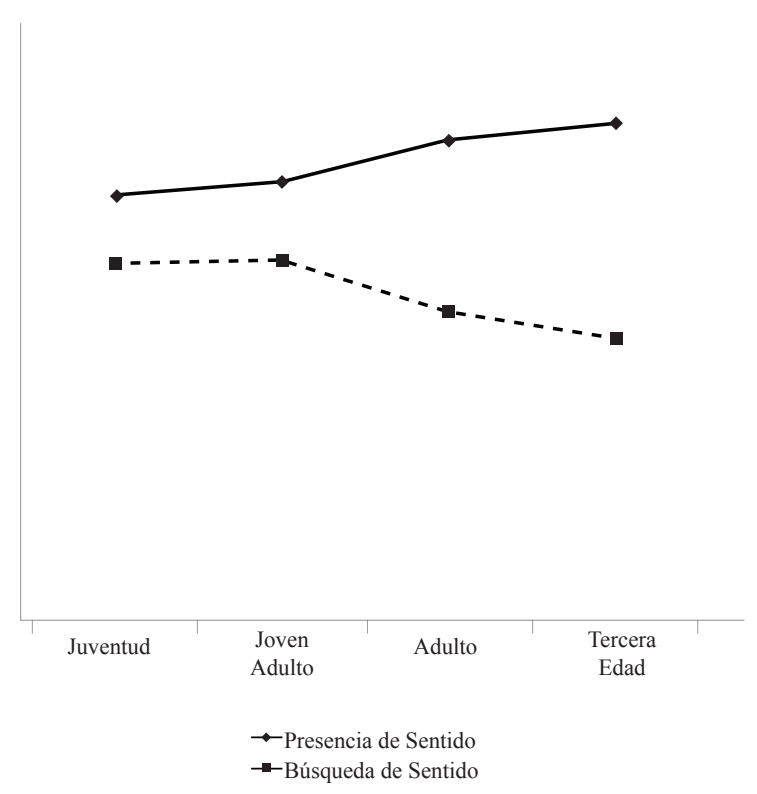

Figura 1. Medias de los scores de presencia y búsqueda de sentido en función del ciclo de la vida

La prueba post hoc de Bonferroni indicó diferencias de los promedios en presencia de sentido para los grupos jóvenes $(M=25,6, D T=6,71)$ y adultos $(M=28,8, D T=4,96)$, jóvenes y tercera edad $(M=29,7, D T=3,36)$, adultos y jóvenes adultos $(M=26,3, D T=6,47)$, y, por fin, jóvenes adultos y tercera edad. Por otra parte, con respecto a la dimensión búsqueda de sentido, el mismo test indicó diferencias entre los promedios de las siguientes fases de la vida: jóvenes $(M=21,4, D T$ $=7,79)$ y adultos $(M=18,5, D T=7,50)$, jóvenes y tercera edad $(M=17,0, D T=5,97)$, jóvenes adultos $(M=21,8, D T=8,12)$ y adultos $\mathrm{y}$, finalmente, jóvenes adultos y tercera edad.

Para conocer las diferencias entre los promedios de los puntajes del pasado, presente y futuro, según las fases de la vida, se realizó un análisis de varianza (Anova). En el caso, han sido observadas diferencias en las tres dimensiones de tiempo: pasado $(F=4,56, p<0,01)$, presente $(F=5,77, p<$ $0,001)$ y futuro $(F=33,83, p<0,001)$. En la figura 2 se presenta la percepción temporal en función de las fases de la vida.

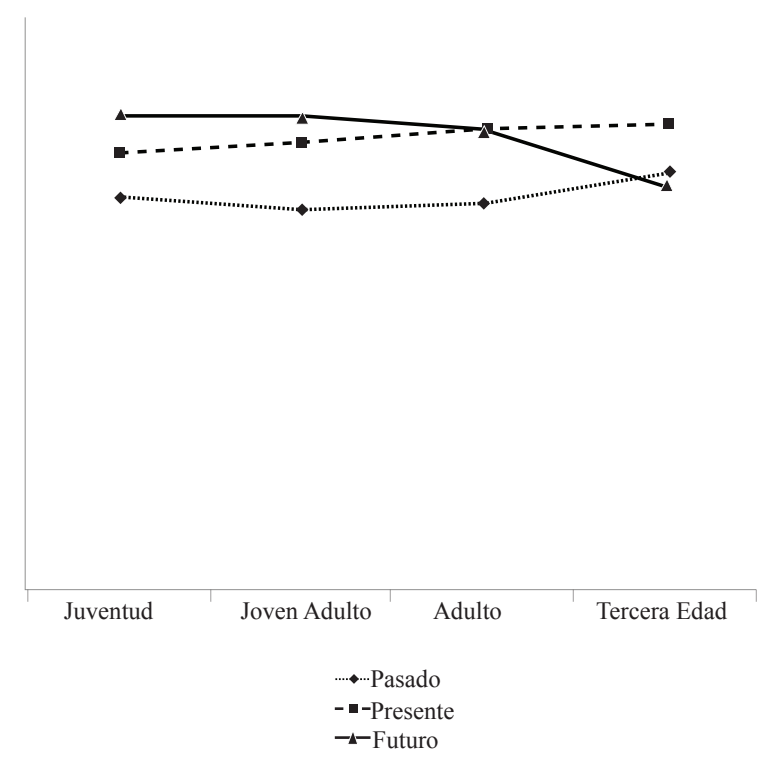

Figura 2. Representación gráfica del desarrollo de la percepción temporal en función de las fases de la vida.

El test de Bonferroni señala dónde exactamente existen diferencias en los puntajes promedios de las percepciones temporales. En el pasado, se observó diferencia entre jóvenes adultos $(M=10,5, D T=$ $2,42)$ y tercera edad $(M=11,5, D T=2,01)$; en el presente las diferencias han sido entre jóvenes $(M$ $=12,1, D T=2,20)$ y adultos $(M=12,8, D T=1,57)$, y jóvenes y tercera edad $(M=12,9, D T=1,72)$. Por fin, respecto al factor futuro, se comprobaron diferencias entre la tercera edad $(M=11,2, D T=$ $1,72)$ y los siguientes grupos: jóvenes $(M=13,2$, $D T=1,83)$, jóvenes adultos $(M=13,2, D T=1,84)$ y adultos $(M=12,7, D T=1,95)$.

La tabla 1 presenta las correlaciones entre el índice de congruencia (Al mirar mi vida en general: admito que hay una gran distancia entre quien "soy" y quien "podría ser") y las percepciones temporales en cada fase de la vida. Los resultados muestran que en los grupos de jóvenes y jóvenes adultos se asocian negativamente con las percepciones del pasado, presente y futuro. En el grupo de adultos estas correlaciones se mantuvieron solamente con respecto al pasado y presente. No obstante, en la 
tercera edad la asociación fue positiva únicamente con el futuro.

Tabla 1

Correlaciones entre las percepciones del tiempo y el indice de congruencia en las fases de la vida

\begin{tabular}{lcccc}
\hline & Pasado & Presente & futuro \\
\hline & Juventud & $-0,23^{* * *}$ & $-0,34^{* * *}$ & $-0,24 * * *$ \\
Índice de & $\begin{array}{l}\text { Joven } \\
\text { adulto }\end{array}$ & $-0,31^{* * *}$ & $-0,35^{* * *}$ & $-0,28^{* * *}$ \\
& Adulto & $-0,19^{*}$ & $-0,20^{* *}$ & $-0,01$ \\
& Vejez & 0,01 & 0,07 & $0,20^{*}$ \\
\hline
\end{tabular}

$* p=0,05, * * p<0,05, * * * p<0,0001$

\section{Discusión}

El objetivo principal de esta investigación ha sido conocer en qué medida la percepción ontológica del tiempo y la sensación de presencia y búsqueda de sentido cambian según el ciclo de vida. De acuerdo con los hallazgos, se entiende que este objetivo ha sido alcanzado. No obstante, es necesario reconocer potenciales limitaciones del estudio, pues se contó con una muestra de conveniencia, que no representa a toda la población brasileña, así como hubo una desproporcionalidad de personas con respecto a los grupos de edad. De este modo, es posible que los resultados no puedan ser generalizados más allá de este estudio. Sin embargo, hay que señalar que este ha sido uno de los esfuerzos aislados para tratar la percepción temporal desde la perspectiva de la logoterapia, y así se confía ofrecer una contribución al área. Aunque Frankl (1988, 1989, 1992) enfatizó en sus publicaciones en la relación entre la transitoriedad de la existencia y el sentido de la vida, pocas evidencias empíricas se han reunido al respecto.

En relación con la primera pregunta, “¿Cuál es el grado de presencia y búsqueda de sentido en las fases del desarrollo humano?", se ha observado que la percepción de presencia de sentido suele aumentar según incrementan los rangos de edad, en cuanto que la búsqueda disminuye con el pasar de los años, corroborando la teoría ontológica del tiempo (Frankl, 1988). La búsqueda presupone que el individuo no encontró el sentido, así en edades tempranas las personas perciben menos realizaciones, ya que las posibilidades se encuentran en el futuro. En contraposición, las personas de la tercera edad, que supuestamente transformaran sus posibilidades en realidades, presentan mayores promedios de realizaciones y menores de búsqueda de sentido.

Este estudio es consonante con el de Lukas (1986), quien constató que los jóvenes entre 15 y 30 años no encuentran los valores más elevados, es decir, perciben menos sentido en la vida, cuando son comparados con las personas entre 40 y 60 años. En el caso, la mayor aspiración de los jóvenes es tan solo descubrir el significado existencial de sus vidas, lo que evidencia una capacidad menor de encontrar sentidos (Lukas, 1990a). Con respecto a la perspectiva temporal, esta autora comprende que la "vivencia de falta de futuro" puede caracterizar a jóvenes y adultos, lo que ocasiona una supuesta falta de sentido en el futuro, que puede implicar en dejar de dar una respuesta en el presente (Lukas, 1990a, 1990b).

Según Frankl (1992), la biografía se concluye solamente en la muerte, cuando se pierde totalmente el futuro. De este modo, el valor del ser humano reside justamente en el pasado, ya que se encuentran en esta dimensión todas las realizaciones humanas (valores vivenciales, creativos y actitudinales). Solamente la contemplación de sentido es más plena en las fases maduras de la existencia.

La segunda pregunta de este estudio fue "¿Cómo las personas perciben la temporalidad (presente, pasado y futuro) en las fases de la vida?". La perspectiva del pasado como algo estructurante de la persona declina entre los jóvenes adultos y en la tercera edad. Hay aquí una nueva preocupación, una consciencia más clara de la finitud humana. Así, en las edades maduras el presente se torna 
imperativo, puesto que en el momento presente el hombre se encuentra confrontado con los valores, que necesitan ser logrados, y los sentidos se encuentran en las situaciones de la vida que se presenta (Frank1, 2003a). En la tercera edad, el futuro se torna más restricto, en comparación con las demás fases de la vida, corroborando las concepciones de Frankl (1988) y Lukas (1992) cuando señalan que las posibilidades se restringen en los ancianos, pero el pasado se constituye de un área amplia, puesto que ya lograron gran parte de su existencia, cambiando las posibilidades (futuro) para la realidad (pasado).

Ramovš (2009) comprende que en la cultura occidental falta la creencia de que la tercera edad también es un periodo significativo, equiparable a los demás periodos de la vida. A propósito, advierte este autor que en los días actuales las personas deben asumir una actitud de su propio pasado, presente y futuro, fundamentalmente en la tercera edad. En esta dirección, el estudio de Vieira (2014), considerando una muestra de adultos mayores, constató que la vitalidad subjetiva está asociada con la mirada positiva del pasado, presente y futuro. En esta dirección, Levy, Slade, Kunkel y Kasl (2002) sugieren que una autopercepción positiva del envejecimiento está relacionada directamente con la longevidad. Desde el punto de vista de la logoterapia, tener un sentido en la vida es una condición necesaria para una actitud positiva en las fases de la vida, incluso en la tercera edad, por lo que se puede presumir que la percepción ontológica del tiempo desempeña un papel relevante en esta evaluación.

Otra variable considerada en el presente estudio fue la congruencia entre el "ser" y el "poder ser". Concretamente, se constató que el índice de congruencia (Al mirar mi vida en general: admito que hay una gran distancia entre quien "soy" y quien "podría ser"), se asoció inversamente con la percepción positiva de la temporalidad solamente entre los jóvenes y jóvenes adultos. Si, por un lado, el joven está por construir su proyecto de vida, el adulto joven se está estabilizando profesionalmente y/o constituyendo familia. Teniendo en cuenta a los adultos, la asociación ocurre solamente en el pasado y el presente. Quizá eso explique que su sensación de realización es más nítida en el presente, en la realización de su deber y los logros de sentido que se encuentran en el pasado.

En la tercera edad solamente una dimensión temporal (futuro) se asoció de forma positiva con el índice de congruencia. De este modo, al contemplar posibilidades de ser en el mundo, las personas mayores perciben motivos para estar en el mundo, lo que representa una tensión existencial y posibles motivos y razones para vivir. Además, esa tensión es fundamental para la salud y el bienestar psíquico del ser humano (Frankl, 1990). Por otro lado, cuantas menos posibilidades de realizaciones perciben, menor la distancia entre su ser y el poder ser. Esta consciencia de percibir el futuro también está relacionada con la consciencia de la muerte, precisamente en las fases finales de la vida (Lukas, 1992).

En la logoterapia, la muerte y la conciencia de la finitud son fundamentales para realizar sentido en la vida, puesto que pone en movimiento la tensión entre el ser y el poder ser. Frankl (1989, 2003a) concibe que la muerte da sentido a la vida, en la medida en que el hombre percibe un límite para construir su ser en el mundo. Por esta razón, en la tercera edad, ya que hay pocas posibilidades de cambiar su ser, aumenta la percepción de la distancia entre quien uno es y quien podría ser

\section{Implicaciones clínicas}

Esta investigación ha constatado que las variables presencia de sentido, búsqueda de sentido y percepción ontológica del tiempo cambian según el ciclo de vida, de modo que es posible realizar algunas reflexiones prácticas en el ámbito clínico. Primeramente, de acuerdo con Frankl (2001), el hombre actual sufre por el sentimiento del absurdo acompañado por una sensación de vacío existencial 
que compromete su bienestar. Esta frustración puede ser trabajada en la medida en que se cuestione el sentido en la temporalidad, investigando posibilidades concretas y reales. Como se ha indicado anteriormente, la voluntad de sentido puede encontrarse en estado de latencia en cualquier fase de la vida, resultando en una sensación de vacío existencial. Considerando los instrumentos aquí utilizados, es posible, en el contexto clínico, identificar en qué dirección se encuentra la percepción de sentido del paciente.

A partir de los resultados de esta investigación, también es posible pensar en programas de promoción del sentido para prevenir el vacío existencial de forma específica para cada fase de la vida. Por ejemplo, en la adolescencia es fundamental trabajar la perspectiva del futuro y el proyecto de vida. De este modo, es necesario también despertar la consciencia y la responsabilidad humana en sus opciones en el presente, puesto que el sentido fluye desde el futuro hacia el pasado. En el adulto, se podría poner énfasis en el presente y sus respectivos sentidos y responsabilidades. Ya en las personas de la tercera edad, se hace imperativo conocer las posibilidades futuras y las tareas concretas que ofrezcan sentido. Este intento se justifica en la medida en que la voluntad de vivir y lograr objetivos mejora el estado afectivo, y, por lo tanto, fortalece el sistema inmunológico (Lukas, 2011).

En conclusión, parece evidente la importancia de pensar sobre la búsqueda del sentido en la vida de las personas, considerando la percepción temporal de su existencia. Seguro ello contribuirá para que se encuentre un sentido de la vida más auténtico, que considere desde las realizaciones presentes hasta la finitud. Con una mirada holística temporal de su vida, la persona humana no se contemplará solamente a sí misma, inmersa en el pasado, ni tampoco va a adelantarse inadvertidamente hacia el futuro, pero necesita percibir el pasado para pensar sus elecciones del presente, a fin de cambiar el futuro (posibilidades) para el pasado (realidad), creando su propio ser en el mundo.

\section{Referencias}

Agudo, V. R. C. (2008). A transição para a idade adulta e os seus marcos: Que efeito na sintomatologia depressiva? (Tesis de Maestria (no publicada), Universidade de Lisboa, Portugal).

Aquino, T. A. A. (2009). Atitudes e intenções de cometer o suicídio: Seus correlatos existenciais e normativos. (Tesis Doctoral (no publicada), Universidad Federal de Paraíba, João Pessoa, $\mathrm{PB}$, Brasil).

Aquino, T. A. A., Gouveia, V. V., Aguiar, A. A., Serafim, T. D. B., Pontes, A. M., Pereira, G. A., \& Ferandes, A. S. (2015). Questionário de Sentido de Vida: Evidências de sua validade fatorial e consistência interna. Psicologia: Ciência e Profissão, 35, 4-19.

Barrientos-Parra, J. (2004). O Estatuto da Juventude: Instrumento para o desenvolvimento integral dos jovens. Revista de Informação Legislativa, 41, 131-152.

Erikson, E. (1987). Identidade, juventude e crise. 2. ${ }^{\mathrm{a}}$ Ed. Río de Janeiro: Guanabara.

Erikson, E. (1998). O ciclo de vida completo. Porto Alegre, RS: Artmed.

Frankl, V. E. (1988). La voluntad de sentido. Barcelona: Herder.

Frankl, V. E. (1989). Un sentido para a vida: Psicoterapia e humanismo. Aparecida, SP: Editora Santuario.

Frankl, V. (1990). Logoterapia y análisis existencial. Barcelona: Herder.

Frankl, V. (1992). Psicoanálisis y existencialismo. De la psicoterapia a la logoterapia. 5. ${ }^{\mathrm{a}}$ ed. México: Fondo de Cultura Económica.

Frankl, V. (2000). En el principio era el sentido. Barcelona: Paidós.

Frankl, V. (2001). Teoría y terapia de las neurosis: Iniciación a la logoterapia y análisis existencial. Barcelona: Herder.

Frankl, V. (2003a). El hombre en busca de sentido. Barcelona: Herder. 
Frankl, V. (2003b). El hombre doliente: Fundamentos antropológicos de la psicoterapia. Barcelona: Herder.

Heidegger, M. (1989). El Ser y el Tiempo. 7. ${ }^{\mathrm{a}}$ ed. México/Madrid/Buenos Aires: Fondo de Cultura Económica.

Levinson, D. J. (1986). A conception of adult development. American Psychologist, 41, 3-13.

Levy, B. R., Slade, M. D., Kunkel, S. R., \& Kasl, S. V. (2002). Longevity increased by positive self-perceptions of aging. Journal of Personality and Social Psychology, 2, 261-270.

Lukas, E. (1986). Logo-Test, Test zur Messung von innerer Sinnerfuellung und existentieller Frustration. Viena: Ed. Deuticker.

Lukas, E. (1990a). Assistência logoterapeutica: Transição para uma psicologia humanizada. Petrópolis, RJ: Vozes.

Lukas, E. (1990b). Mentalização e saúde: A arte de viver e logoterapia. Petrópolis, RJ: Vozes.

Lukas, E. (1992). Prevenção psicológica: A prevenção de crises e a proteção do mundo interior do ponto de vista da logoterapia. Petrópolis RJ: Vozes.

Lukas, E. (2011). También tu sufrimiento tiene sentido: Alivio en la crisis a través de la logoterapia. México: Ediciones LAG.

Raffa, L. O., \& Bortolossi, A. I. (2007). Percepção das agências de emprego quanto à recolocação de profissionais maduros no mercado de trabalho.
Psicologia America Latina. n. 9. Recuperado de http://www.ulapsi.org/portal/arquivos/revistas/ Numero-9.pdf

Ramovš, J. (2009). The preventive care significance of Frankl's logotherapy for contemporary programs aimed at quality aging and good intergenerational relations. In A. Batthyany \& J. Levinson (Eds.), Existential psychotherapy of meaning: A handbook of logotherapy and existential analysis (pp. 245-275). Phoenix, AZ: Zeig, Tucker \& Theisen, INC.

Soares, S. Carvalho, L. \& Kipns, B. (2003). Os jovens adultos de 18 a 25 anos: Retrato de uma divida da política educacional. Río de Janeiro: IPEA.

Steger, F. M., Frazier, P., Oishi, S., \& Kaler, M. (2006). The meaning in Life Questionnaire: Assessing the presence of and search for meaning in life. Journal of Counseling Psychology, 53, 80-93.

Vieira, D. C. R. (2014). A velhice em uma dimensão existencial: Perspectiva entre sentido da vida, religiosidade, vitalidade e temporalidade. (Tesis de Maestría (no publicada), Universidad Federal de Paraíba, João Pessoa, PB, Brasil).

World Health Organization (1986). Young people's health - A challenge for society. Report of a WHO Study Group on Young People and Health for All. Technical Report Series 731. Geneva: WHO.

\section{Recibido 07 de abril de 2015 Aprobado: 17 de agosto de 2016}




\section{Apéndice}

Escala de percepción ontológica del tiempo (ЕРОТ)

Instrucciones: para cada afirmación sobre el pasado, el presente y el futuro, indique el grado de concordancia/discordancia que esté más de acuerdo con su experiencia personal:

\begin{tabular}{|c|c|c|c|c|c|}
\hline & $\begin{array}{c}\text { Totalmente } \\
\text { en } \\
\text { desacuerdo }\end{array}$ & En desacuerdo & $\begin{array}{l}\text { Ni en acuerdo ni } \\
\text { en desacuerdo }\end{array}$ & De acuerdo & $\begin{array}{l}\text { Totalmente } \\
\text { de acuerdo }\end{array}$ \\
\hline \multicolumn{6}{|l|}{ Al mirar el pasado: } \\
\hline $\begin{array}{l}\text { 1. Me siento realizado(a) por lo que ya he } \\
\text { logrado en la vida. }\end{array}$ & 1 & 2 & 3 & 4 & 5 \\
\hline $\begin{array}{l}\text { 2. Percibo que tengo evolucionado para la } \\
\text { persona que siempre he querido ser. }\end{array}$ & 1 & 2 & 3 & 4 & 5 \\
\hline 3. Haría todo otra vez. & 1 & 2 & 3 & 4 & 5 \\
\hline \multicolumn{6}{|l|}{ Al mirar el presente: } \\
\hline $\begin{array}{l}\text { 4. Veo siempre una razón para estar en el } \\
\text { mundo. }\end{array}$ & 1 & 2 & 3 & 4 & 5 \\
\hline $\begin{array}{l}\text { 5. Creo que estoy realizando tareas signifi- } \\
\text { cativas. }\end{array}$ & 1 & 2 & 3 & 4 & 5 \\
\hline $\begin{array}{l}\text { 6. Encuentro siempre una razón para dejar la } \\
\text { cama por la mañana. }\end{array}$ & 1 & 2 & 3 & 4 & 5 \\
\hline \multicolumn{6}{|l|}{ Al mirar el futuro: } \\
\hline 7. Veo muchas posibilidades de elección. & 1 & 2 & 3 & 4 & 5 \\
\hline 8. Percibo una razón por la cual vivir. & 1 & 2 & 3 & 4 & 5 \\
\hline $\begin{array}{l}\text { 9. Veo que tengo un ideal o sueño a ser rea- } \\
\text { lizado. }\end{array}$ & 1 & 2 & 3 & 4 & 5 \\
\hline \multicolumn{6}{|l|}{ Al mirar mi vida en general: } \\
\hline $\begin{array}{l}\text { 10. Admito que hay una gran distancia entre } \\
\text { quien "soy" y quien "podría ser". }\end{array}$ & 1 & 2 & 3 & 4 & 5 \\
\hline
\end{tabular}

